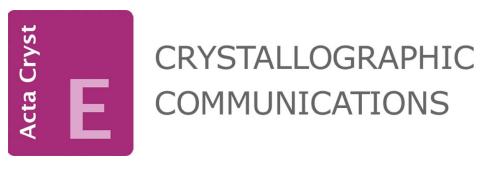

ISSN 2056-9890

Received 21 January 2018

Accepted 21 January 2018

Edited by V. Khrustalev, Russian Academy of Sciences, Russia

Keywords: crystal structure; natural alkaloids; azoninoindoles; Alzheimer disease; synchrotron radiation.

CCDC reference: 1818381

Supporting information: this article has supporting information at journals.iucr.org/e

\section{Design of new anti-Alzheimer drugs: ring-expansion synthesis and synchrotron X-ray diffraction study of dimethyl 4-ethyl-11-fluoro-1,4,5,6,7,8-hexahydro- azonino[5,6-b] indole-2,3-dicarboxylate}

\author{
Flavien A. A. Toze, ${ }^{a *}$ Anna V. Listratova, ${ }^{b}$ Leonid G. Voskressensky, ${ }^{b}$ Natalia Yu. \\ Chernikova, ${ }^{\text {c Nikolai N. Lobanov, }}$, Alexey N. Bilyachenko ${ }^{\text {d }}$ and Pavel V. \\ Dorovatovskii $^{\mathrm{e}}$
}

\begin{abstract}
${ }^{a}$ Department of Chemistry, Faculty of Sciences, University of Douala, PO Box 24157, Douala, Republic of , Cameroon, bOrganic Chemistry Department, Peoples' Friendship University of Russia, 6 Miklukho-Maklaya St., Moscow 117198, Russian Federation, 'Chemistry and Biology Department, Peoples' Friendship University of Russia, 6 Miklukho-Maklay St., Moscow 117198, Russian Federation, 'd Inorganic Chemistry Department, Peoples' Friendship University of Russia, 6 Miklukho-Maklay St., Moscow 117198, Russian Federation, and 'National Research Centre "Kurchatov Institute", 1 Acad. Kurchatov Sq., Moscow 123182, Russian Federation. *Correspondence e-mail: toflavien@yahoo.fr
\end{abstract}

The title compound, $\mathrm{C}_{20} \mathrm{H}_{23} \mathrm{FN}_{2} \mathrm{O}_{4}$, is the product of a ring-expansion reaction from a seven-membered fluorinated hexahydroazepine to a nine-membered azonine. The nine-membered azonine ring of the molecule adopts a chair-boat conformation. The $\mathrm{C}=\mathrm{C}$ and $\mathrm{C}-\mathrm{N}$ bond lengths $[1.366$ (3) and 1.407 (3) $\AA$, respectively] indicate the presence of conjugation within the enamine $\mathrm{CH}_{2}-$ $\mathrm{C}=\mathrm{C}-\mathrm{N}-\mathrm{CH}_{2}$ fragment. The substituent planes at the $\mathrm{C}=\mathrm{C}$ double bond of this fragment are twisted by $16.0(3)^{\circ}$ as a result of steric effects. The amine $\mathrm{N}(\mathrm{Et}) \mathrm{N}$ atom has a trigonal-pyramidal configuration (sum of the bond angles = $\left.346.3^{\circ}\right)$. The interplanar angle between the two carboxylate substituents is $60.39(8)^{\circ}$. In the crystal, molecules form zigzag chains along [010] by intermolecular $\mathrm{N}-\mathrm{H} \cdots \mathrm{O}$ hydrogen-bonding interactions, which are further packed in stacks toward [100]. The title azoninoindole might be considered as a candidate for the design of new Alzheimer drugs.

\section{Chemical context}

Eight-, nine-, and ten-membered heterocycles, often referred to as medium-sized rings, remain largely unexplored because of the lack of general convenient routes for their synthesis. Meanwhile, such medium-sized heterocycles, in particular azonine, frequently occur in natural products, such as alkaloids (Neuss et al., 1959, 1962; Uprety \& Bhakuni, 1975), and thus they are considered to be promising fragments in drug design.

Voskressensky and his group have pioneered the tandem transformation of fused tetrahydropyridines into azines bearing an enamine moiety in the eight-membered ring under the action of activated alkynes. Based on this reaction, convenient preparative routes to tetrahydropyrrolo[2,3-d]azocines (Varlamov et al., 2002), tetrahydroazocino[5,4-b]indoles, and tetrahydroazocino[4,5- $b$ ]indoles (Voskressensky et al., 2004) have been elaborated. The application of a similar approach to hexahydroazepine gives rise to azoninoindoles (Nguyen et al., 2017), which are otherwise hard to obtain.

Azoninoindole I was successfully synthesized from the initial 2-ethyl-9-fluoro-1,2,3,4,5,6-hexahydroazepino[4,3- $b$ ]indole via a domino reaction under the action of dimethyl 


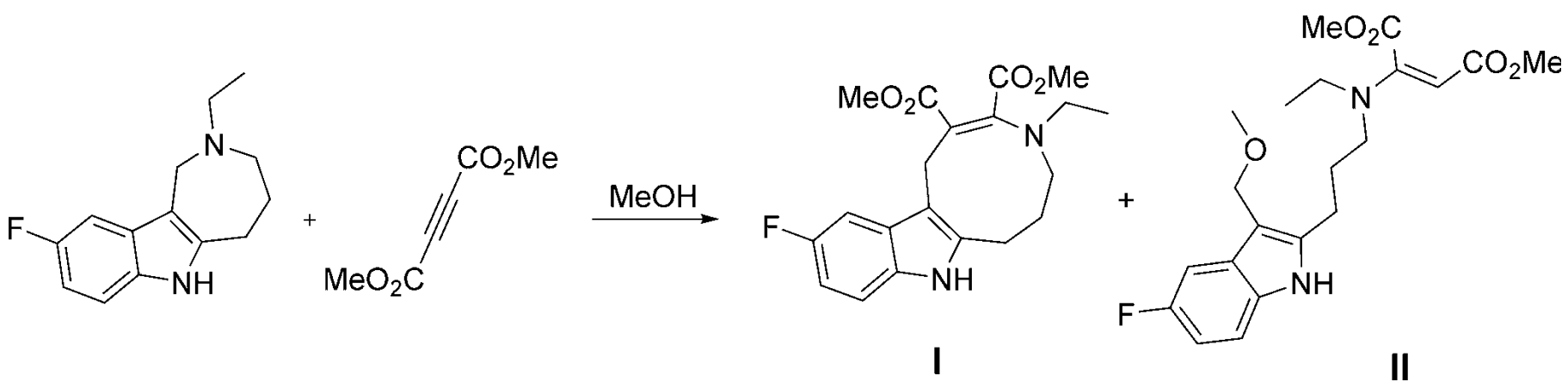

Figure 1

The synthesis of dimethyl 4-ethyl-11-fluoro-1,4,5,6,7,8-hexahydroazonino[5,6- $b$ indole-2,3-dicarboxylate I in methanol.

acetylenedicarboxylate in methanol at room temperature (Fig. 1). The domino reaction results in the expansion of the hexahydroazepine ring to the azonine viz. dimethyl 4-ethyl11-fluoro-1,4,5,6,7,8-hexahydroazonino[5,6- $b$ ]indole-2,3dicarboxylate (I). 3-Methoxymethyl-substituted indole II was isolated as a by-product of this reaction.

The azonine systems, as a result of their specific structure, are known to act as ligands towards different receptors, thus demonstrating diverse types of biological activity (Magnus et al., 1987; Kuehne, Bornman et al., 2003; Kuehne, He et al., 2003; Afsah et al., 2009; Rostom, 2010; Tanaka et al., 2014; Soldi et al., 2015; Hartman \& Kuduk, 2016), including antiAlzheimer's disease activity (Nguyen et al., 2017).

The title compound $\mathbf{I}, \mathrm{C}_{20} \mathrm{H}_{23} \mathrm{FN}_{2} \mathrm{O}_{4}$, is the product of a ring expansion reaction from a seven-membered fluorinated hexahydroazepine to a nine-membered azonine. The molecular structure of $\mathbf{I}$ is unambiguously confirmed by the X-ray diffraction study (Fig. 2).

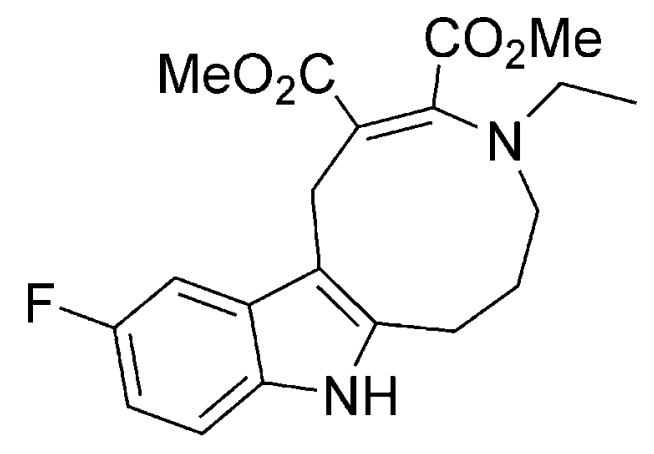

\section{Structural commentary}

Compound $\mathbf{I}$ is isostructural to the non-fluorinated analog published by us very recently (Nguyen et al., 2017). The ninemembered azonine ring of the molecule adopts a chair-boat conformation (the basal planes are $\mathrm{N} 4-\mathrm{C} 5 / \mathrm{C} 1-\mathrm{C} 12 B$ and $\mathrm{C5}-$ $\mathrm{C} 6 / \mathrm{C} 7 A-\mathrm{C} 12 B$, respectively). It should be noted that the analogous nine-membered azonine ring in the related compound methyl 4-ethyl-11-methyl-1,4,5,6,7,8-hexahydroazonino[5,6- $b$ ] indole-2-carboxylate adopts a twisted boat conformation (Voskressensky, et al., 2006). The $\mathrm{C} 2=\mathrm{C} 3$ and C3-N4 bond lengths [1.366 (3) and 1.407 (3) A, respectively] indicate the presence of conjugation within the enamine
$\mathrm{C} 2=\mathrm{C} 3-\mathrm{N} 4$ fragment. The substituent planes at the $\mathrm{C} 2=\mathrm{C} 3$ double bond are twisted by $16.0(3)^{\circ}$ because of steric effects. The N4 nitrogen atom has a trigonal-pyramidal configuration (sum of the bond angles is $346.3^{\circ}$ ). The interplanar angle between the two carboxylate substituents is $60.39(8)^{\circ}$.

\section{Supramolecular features}

In the crystal, molecules of $\mathbf{I}$ form zigzag chains along [010] by intermolecular $\mathrm{N}-\mathrm{H} \cdots \mathrm{O}^{\mathrm{i}}$ hydrogen-bonding interactions (Table 1, Fig. 3), which are further packed in stacks towards [100].

\section{Synthesis and crystallization}

Dimethyl acetylenedicarboxylate (170 $\mathrm{mg}, 1.2 \mathrm{mmol})$ was added to 2-ethyl-9-fluoro-1,2,3,4,5,6-hexahydroazepino[4,3- $b$ ] indole $(232 \mathrm{mg}, 1 \mathrm{mmol})$ dissolved in methanol $(10 \mathrm{ml})$. The reaction mixture was stirred for $2 \mathrm{~h}$ at room temperature with

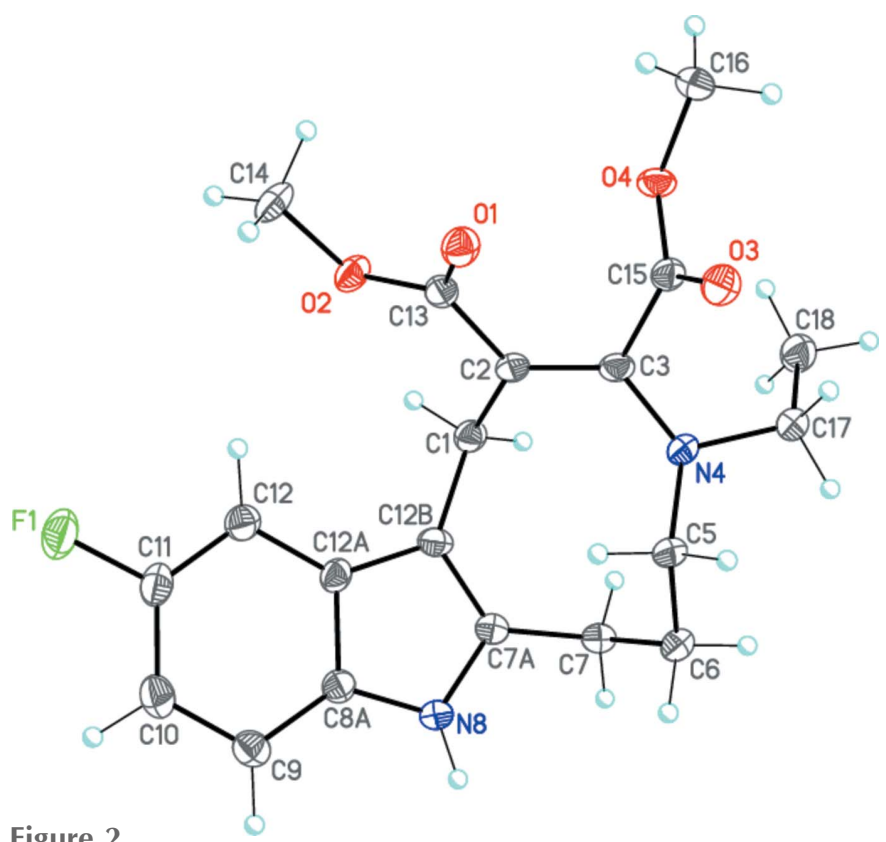

Figure 2

The molecular structure of I. Displacement ellipsoids are drawn at the $50 \%$ probability level. $\mathrm{H}$ atoms are shown as small spheres of arbitrary radius. 
Table 1

Hydrogen-bond geometry $\left(\AA,^{\circ}\right)$.

\begin{tabular}{lllll}
\hline$D-\mathrm{H} \cdots A$ & $D-\mathrm{H}$ & $\mathrm{H} \cdots A$ & $D \cdots A$ & $D-\mathrm{H} \cdots A$ \\
\hline $\mathrm{N} 8-\mathrm{H} 8 \cdots \mathrm{O} 1^{\mathrm{i}}$ & $0.93(3)$ & $2.17(3)$ & $3.025(3)$ & $153(2)$ \\
\hline
\end{tabular}

Symmetry code: (i) $-x+1, y+\frac{1}{2},-z+\frac{1}{2}$.

the TLC real-time control. Then the solvent was removed in vacuo and the residue was chromatographed over silica with ethylacetate:hexane as eluent to yield the target fluorinated azoninoindole I (22\%) and 3-methoxymethylindole II. Lightyellow crystals of azoninoindole I suitable for X-ray crystallographic analysis were grown by slow evaporation of an ethylacetate:hexane (1:1) solution, m.p. 456-458 K.

${ }^{1} \mathrm{H}$ NMR $\left(\mathrm{CDCl}_{3}, \delta / \mathrm{ppm}, J / \mathrm{Hz}\right): 0.98(t, 3 \mathrm{H}, J=7.2$, $\left.\mathrm{CH}_{3} \mathrm{CH}_{2}\right), 1.78\left(m, 2 \mathrm{H}, 6-\mathrm{CH}_{2}\right), 2.74\left(q, 2 \mathrm{H}, J=7.2, \mathrm{CH}_{3} \mathrm{CH}_{2}\right)$, $2.93\left(m, 2 \mathrm{H}, 7-\mathrm{CH}_{2}\right), 3.06\left(m, 2 \mathrm{H}, 5-\mathrm{CH}_{2}\right), 3.96\left(s, 2 \mathrm{H}, 1-\mathrm{CH}_{2}\right)$, $3.74\left(s, 3 \mathrm{H}, \mathrm{CO}_{2} \mathrm{CH}_{3}\right), 3.77\left(s, 3 \mathrm{H}, \mathrm{CO}_{2} \mathrm{CH}_{3}\right), 6.82\left(d d d, 2 \mathrm{H},{ }^{1,3} \mathrm{~J}\right.$ $\left.=9.0,{ }^{1,3} \mathrm{~J}=9.0,{ }^{1,4} \mathrm{~J}=2.3, \mathrm{CH}-\mathrm{Ar}\right), 7.13$ ( $\left.m, 2 \mathrm{H}, \mathrm{CH}-\mathrm{Ar}\right), 7.74$ (br s $1 \mathrm{H}, \mathrm{NH}) .{ }^{13} \mathrm{C} \mathrm{NMR}$ (DMSO- $\left.d_{6}, \delta / \mathrm{ppm}, J / \mathrm{Hz}\right): 15.2\left(\mathrm{CH}_{3}\right)$, $21.9\left(\mathrm{CH}_{2}\right), 23.8\left(\mathrm{CH}_{2}\right), 27.1\left(\mathrm{CH}_{2}\right), 44.5\left(\mathrm{CH}_{2}\right), 52.3\left(\mathrm{CH}_{3}\right)$, $52.3\left(\mathrm{CH}_{3}\right), 55.5(\mathrm{CH} 2), 102.5(d, J=22, \mathrm{CH}), 108.2(d, J=26$, $\mathrm{CH}), 108.6(\mathrm{C}), 111.8(d, J=9, \mathrm{CH}), 122.3(\mathrm{C}), 128.3(\mathrm{C}), 132.2$ (C), 137.9 (C), 151.7 (C), 157.1 ( $d, J=231, \mathrm{C}), 166.4$ (C), 169.3 (C). IR (KBr): $v\left(\mathrm{~cm}^{-1}\right)=1723,3373$. Found (\%): C, 64.16; $\mathrm{H}$, 6.19; N, 7.48. $\mathrm{C}_{20} \mathrm{H}_{23} \mathrm{FN}_{2} \mathrm{O}_{4}$. Calculated (\%): C, 64.46; H, 6.86; $\mathrm{N}$, 7.82. Mass-spectrometry, $\mathrm{m} / z\left[\mathrm{I}_{\mathrm{rel}}(\%)\right]: 374\left[\mathrm{M}^{+}\right](100)$, 345 (20), $315(100), 285(30), 227(10), 198(20), 174(30)$, 161 (30), 148 (10), 58 (40), 45 (10).

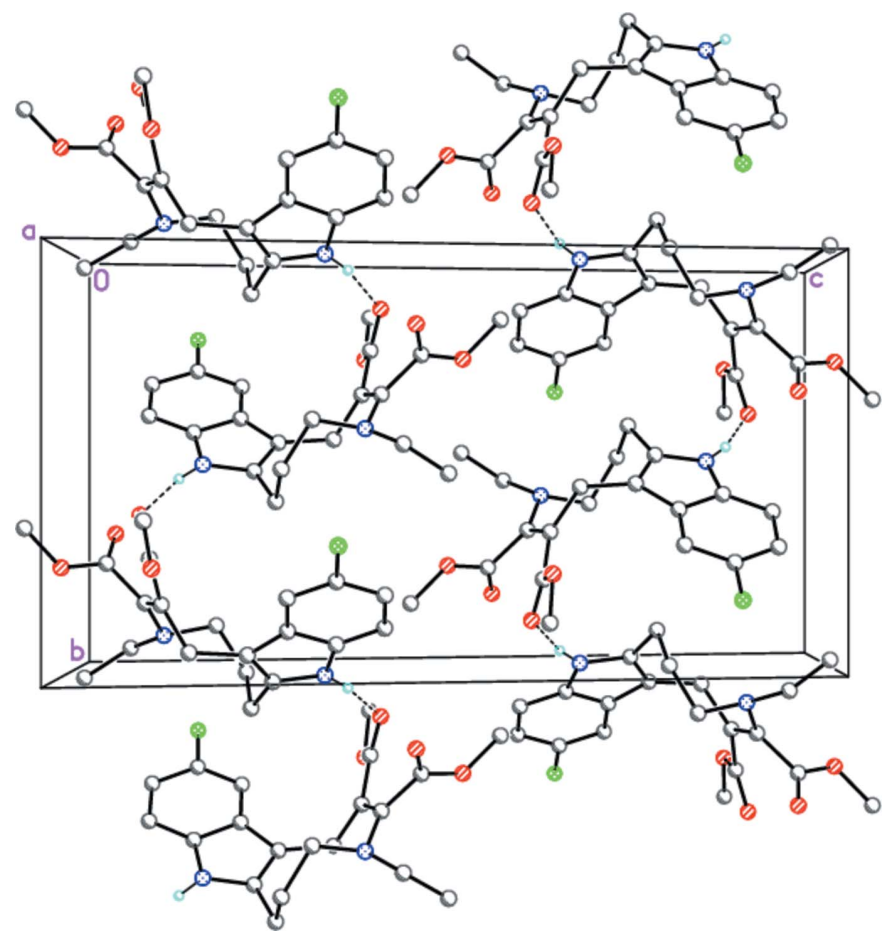

Figure 3

The crystal packing of $\mathbf{I}$ viewed along the $a$-axis direction showing the zigzag chains along [010]. Dashed lines indicate intermolecular $\mathrm{N}-$ $\mathrm{H}$... O hydrogen bonds.
Table 2

Experimental details.

\begin{tabular}{ll}
\hline Crystal data & \\
Chemical formula & $\mathrm{C}_{20} \mathrm{H}_{23} \mathrm{FN}_{2} \mathrm{O}_{4}$ \\
$M_{\mathrm{r}}$ & 374.40 \\
Crystal system, space group & Monoclinic, $P{ }_{1} / c$ \\
Temperature $(\mathrm{K})$ & 100 \\
$a, b, c(\AA)$ & $8.4632(17), 10.993(2), 20.520(4)$ \\
$\beta\left({ }^{\circ}\right.$ & $99.60(3)$ \\
$V\left(\AA^{3}\right)$ & $1882.4(7)$ \\
$Z$ & 4 \\
Radiation type & Synchrotron, $\lambda=0.96990 \AA$ \\
$\mu\left(\mathrm{mm}^{-1}\right)$ & 0.21 \\
Crystal size (mm) & $0.22 \times 0.02 \times 0.02$ \\
& \\
Data collection & \\
Diffractometer & Rayonix SX165 CCD \\
Absorption correction & Multi-scan $(S C A L A ;$ Evans, 2006) \\
$T_{\text {min }}, T_{\text {max }}$ & $0.940,0.980$ \\
No. of measured, independent and & $21117,3850,2463$ \\
$\quad$ observed $[I>2 \sigma(I)]$ reflections & \\
$R_{\text {int }}$ & 0.086 \\
(sin $\theta / \lambda)_{\text {max }}\left(\AA^{-1}\right)$ & 0.640 \\
Refinement & \\
$R\left[F^{2}>2 \sigma\left(F^{2}\right)\right], w R\left(F^{2}\right), S$ & $0.072,0.184,1.01$ \\
No. of reflections & 3850 \\
No. of parameters & 251 \\
H-atom treatment & H atoms treated by a mixture of \\
& independent and constrained \\
$\Delta \rho_{\text {max }}, \Delta \rho_{\text {min }}\left(\mathrm{e} \AA^{-3}\right)$ & refinement \\
\hline & $0.34,-0.43$ \\
\hline &
\end{tabular}

Computer programs: MarCCD (Doyle, 2011), iMOSFLM (Battye et al., 2011), SHELXT (Sheldrick, 2015a), SHELXL2014 (Sheldrick, 2015b) and SHELXTL (Sheldrick, 2008).

\section{Refinement}

Crystal data, data collection and structure refinement details are summarized in Table 2. The X-ray diffraction study was carried out on the "Belok" beamline of the National Research Center "Kurchatov Institute" (Moscow, Russian Federation) using a Rayonix SX165 CCD detector. A total of 360 images were collected using an oscillation range of $1.0^{\circ}$ ( $\varphi$ scan mode, two different crystal orientations) and corrected for absorption using the SCALA program (Evans, 2006). The data were indexed, integrated and scaled using the utility $i$ MOSFLM in the CCP4 program suite (Battye et al., 2011).

The hydrogen atoms of the amino groups were localized in the difference-Fourier map and refined isotropically with fixed displacement parameters $\left[U_{\text {iso }}(\mathrm{H})=1.2 U_{\text {eq }}(\mathrm{N})\right]$. The other hydrogen atoms were placed in calculated positions with $\mathrm{C}-$ $\mathrm{H}=0.95-0.99 \AA$ and refined in the riding model with fixed isotropic displacement parameters $\left[U_{\text {iso }}(\mathrm{H})=1.2 U_{\text {eq }}(\mathrm{C})\right]$.

\section{Funding information}

This work was supported by the RUDN Program "5-100". Xray crystallographic studies using synchrotron radiation were performed at the unique scientific facility Kurchatov Synchrotron Radiation Source supported by the Ministry of Education and Science of the Russian Federation (project code RFMEFI61917X0007). 


\section{References}

Afsah, E. M., Fadda, A. A., Bondock, S. \& Hammouda, M. M. (2009). Z. Naturforsch. Teil B, 64, 415-422.

Battye, T. G. G., Kontogiannis, L., Johnson, O., Powell, H. R. \& Leslie, A. G. W. (2011). Acta Cryst. D67, 271-281.

Doyle, R. A. (2011). MarCCD software manual. Rayonix L. L. C. Evanston, IL 60201 USA.

Evans, P. (2006). Acta Cryst. D62, 72-82.

Hartman, G. D. \& Kuduk, S. (2016). Patent US2016/272599A1. Novira Therapeutics, Inc., USA.

Kuehne, M. E., Bornmann, W. G., Markó, I., Qin, Y., LeBoulluec, K. L., Frasier, D. A., Xu, F., Mulamba, T., Ensinger, C. L., Borman, L. S., Huot, A. E., Exon, C., Bizzarro, F. T., Cheung, J. B. \& Bane, S. L. (2003). Org. Biomol. Chem. 1, 2120-2136.

Kuehne, M., He, L., Jokiel, P., Pace, C. J., Fleck, M. W., Maisonneuve, I. M., Glick, S. D. \& Bidlack, J. M. (2003). J. Med. Chem. 46, 27162730.

Magnus, P., Ladlow, M. \& Elliott, J. (1987). J. Am. Chem. Soc. 109, 7929-7930.

Neuss, N., Gorman, M., Boaz, H. E. \& Cone, N. J. (1962). J. Am. Chem. Soc. 84, 1509-1510.

Neuss, N., Gorman, M., Svoboda, G. H., Maciak, G. \& Beer, C. T. (1959). J. Am. Chem. Soc. 81, 4754-4755.
Nguyen, V. T., Sorokina, E. A., Listratova, A. V., Voskressensky, L. G., Lobanov, N. N., Dorovatovskii, P. V., Zubavichus, Y. V. \& Khrustalev, V. N. (2017). Acta Cryst. E73, 338-340.

Rostom, S. A. F. (2010). Arch. Pharm. Chem. Life Sci. 343, 73-80.

Sheldrick, G. M. (2008). Acta Cryst. A64, 112-122.

Sheldrick, G. M. (2015a). Acta Cryst. A71, 3-8.

Sheldrick, G. M. (2015b). Acta Cryst. C71, 3-8.

Soldi, R., Horrigan, S. K., Cholody, M. W., Padia, J., Sorna, V., Bearss, J., Gilcrease, G., Bhalla, K., Verma, A., Vankayalapati, H. \& Sharma, S. (2015). J. Med. Chem. 58, 5854-5862.

Tanaka, Y., Gamo, K., Oyama, T., Ohashi, M., Waki, M., Matsuno, K., Matsuura, N., Tokiwa, H. \& Miyachi, H. (2014). Bioorg. Med. Chem. Lett. 24, 4001-4005.

Uprety, H. \& Bhakuni, D. S. (1975). Tetrahedron Lett. 16, 12011204.

Varlamov, A. V., Borisova, T. N., Voskressensky, L. G., Soklakova, T. A., Kulikova, L. N., Chernyshev, A. V. \& Alexandrov, G. G. (2002). Tetrahedron Lett. 43, 6767-6769.

Voskressensky, L. G., Akbulatov, S. V., Borisova, T. N. \& Varlamov, A. V. (2006). Tetrahedron, 62, 12392-12397.

Voskressensky, L. G., Borisova, T. N., Kulikova, L. N., Varlamov, A. V., Catto, M., Altomare, C. \& Carotti, A. (2004). Eur. J. Org. Chem. pp. 3128-3135. 


\section{supporting information}

Acta Cryst. (2018). E74, 298-301 [https://doi.org/10.1107/S2056989018001329]

Design of new anti-Alzheimer drugs: ring-expansion synthesis and synchrotron X-ray diffraction study of dimethyl 4-ethyl-11-fluoro-1,4,5,6,7,8-hexahydroazonino[5,6-b]indole-2,3-dicarboxylate

Flavien A. A. Toze, Anna V. Listratova, Leonid G. Voskressensky, Natalia Yu. Chernikova, Nikolai

N. Lobanov, Alexey N. Bilyachenko and Pavel V. Dorovatovskii

Computing details

Data collection: MarCCD (Doyle, 2011); cell refinement: iMOSFLM (Battye et al., 2011); data reduction: iMOSFLM

(Battye et al., 2011); program(s) used to solve structure: SHELXT (Sheldrick, 2015a); program(s) used to refine structure: SHELXL2014 (Sheldrick, 2015b); molecular graphics: SHELXTL (Sheldrick, 2008); software used to prepare material for publication: SHELXTL (Sheldrick, 2008).

Dimethyl 4-ethyl-11-fluoro-1,4,5,6,7,8-hexahydroazonino[5,6-b]indole-2,3-dicarboxylate

Crystal data

$\mathrm{C}_{20} \mathrm{H}_{23} \mathrm{FN}_{2} \mathrm{O}_{4}$

$M_{r}=374.40$

Monoclinic, $P 2{ }_{1} / c$

$a=8.4632(17) \AA$

$b=10.993(2) \AA$

$c=20.520(4) \AA$

$\beta=99.60(3)^{\circ}$

$V=1882.4(7) \AA^{3}$

$Z=4$

Data collection

Rayonix SX165 CCD

diffractometer

/f scan

Absorption correction: multi-scan

(SCALA; Evans, 2006)

$T_{\min }=0.940, T_{\max }=0.980$

21117 measured reflections

\section{Refinement}

Refinement on $F^{2}$

Least-squares matrix: full

$R\left[F^{2}>2 \sigma\left(F^{2}\right)\right]=0.072$

$w R\left(F^{2}\right)=0.184$

$S=1.01$

3850 reflections

251 parameters
$F(000)=792$

$D_{\mathrm{x}}=1.321 \mathrm{Mg} \mathrm{m}^{-3}$

Synchrotron radiation, $\lambda=0.96990 \AA$

Cell parameters from 600 reflections

$\theta=3.3-33.0^{\circ}$

$\mu=0.21 \mathrm{~mm}^{-1}$

$T=100 \mathrm{~K}$

Needle, yellow

$0.22 \times 0.02 \times 0.02 \mathrm{~mm}$

3850 independent reflections

2463 reflections with $I>2 \sigma(I)$

$R_{\text {int }}=0.086$

$\theta_{\max }=38.4^{\circ}, \theta_{\min }=3.3^{\circ}$

$h=-10 \rightarrow 10$

$k=-12 \rightarrow 10$

$l=-26 \rightarrow 25$

0 restraints

Primary atom site location: difference Fourier map

Secondary atom site location: difference Fourier map

Hydrogen site location: mixed 
$\mathrm{H}$ atoms treated by a mixture of independent and constrained refinement

$w=1 /\left[\sigma^{2}\left(F_{\mathrm{o}}^{2}\right)+(0.090 P)^{2}\right]$

where $P=\left(F_{\mathrm{o}}^{2}+2 F_{\mathrm{c}}^{2}\right) / 3$

$(\Delta / \sigma)_{\max }<0.001$
$\Delta \rho_{\max }=0.34$ e $\AA^{-3}$

$\Delta \rho_{\min }=-0.43$ e $\AA^{-3}$

Extinction correction: SHELXL, $\mathrm{Fc}^{*}=\mathrm{kFc}\left[1+0.001 \times \mathrm{Fc}^{2} \lambda^{3} / \sin (2 \theta)\right]^{-1 / 4}$

Extinction coefficient: 0.016 (2)

Special details

Geometry. All esds (except the esd in the dihedral angle between two 1.s. planes) are estimated using the full covariance matrix. The cell esds are taken into account individually in the estimation of esds in distances, angles and torsion angles; correlations between esds in cell parameters are only used when they are defined by crystal symmetry. An approximate (isotropic) treatment of cell esds is used for estimating esds involving l.s. planes.

Fractional atomic coordinates and isotropic or equivalent isotropic displacement parameters $\left(\AA^{2}\right)$

\begin{tabular}{|c|c|c|c|c|}
\hline & $x$ & $y$ & $z$ & $U_{\text {iso }} * / U_{\text {eq }}$ \\
\hline $\mathrm{F} 1$ & $-0.11330(17)$ & $0.18565(15)$ & $0.14176(8)$ & $0.0428(5)$ \\
\hline $\mathrm{O} 1$ & $0.35878(18)$ & $0.12235(17)$ & $0.40043(8)$ & $0.0254(5)$ \\
\hline $\mathrm{O} 2$ & $0.13856(17)$ & $0.24026(16)$ & $0.37589(8)$ & $0.0256(5)$ \\
\hline $\mathrm{O} 3$ & $0.72767(18)$ & $0.17340(16)$ & $0.45020(8)$ & $0.0265(5)$ \\
\hline $\mathrm{O} 4$ & $0.54751(18)$ & $0.24913(16)$ & $0.50997(7)$ & $0.0245(5)$ \\
\hline $\mathrm{C} 1$ & 0.3100 & $0.4423(2)$ & $0.33722(11)$ & $0.0220(6)$ \\
\hline $\mathrm{H} 1 \mathrm{~A}$ & 0.1961 & 0.4424 & 0.3430 & $0.026^{*}$ \\
\hline H1B & 0.3592 & 0.5174 & 0.3581 & $0.026^{*}$ \\
\hline $\mathrm{C} 2$ & $0.3917(3)$ & $0.3338(2)$ & $0.37585(11)$ & $0.0197(6)$ \\
\hline $\mathrm{C} 3$ & 0.5500 & $0.3353(2)$ & $0.40360(11)$ & $0.0197(6)$ \\
\hline N4 & $0.6592(2)$ & 0.41914 (19) & $0.38442(9)$ & $0.0215(5)$ \\
\hline $\mathrm{C} 5$ & $0.7014(3)$ & $0.3978(2)$ & $0.31780(11)$ & $0.0225(6)$ \\
\hline $\mathrm{H} 5 \mathrm{~A}$ & 0.6179 & 0.3459 & 0.2920 & $0.027 *$ \\
\hline H5B & 0.8041 & 0.3528 & 0.3229 & $0.027 *$ \\
\hline C6 & $0.7174(3)$ & $0.5148(2)$ & $0.27897(12)$ & $0.0265(6)$ \\
\hline H6A & 0.7461 & 0.4933 & 0.2356 & $0.032 *$ \\
\hline H6B & 0.8060 & 0.5642 & 0.3032 & $0.032 *$ \\
\hline $\mathrm{C} 7$ & $0.5632(3)$ & $0.5921(3)$ & $0.26769(12)$ & $0.0249(6)$ \\
\hline $\mathrm{H} 7 \mathrm{~A}$ & 0.5426 & 0.6237 & 0.3107 & $0.030 *$ \\
\hline H7B & 0.5783 & 0.6625 & 0.2393 & $0.030^{*}$ \\
\hline C7A & $0.4212(3)$ & $0.5196(2)$ & $0.23562(11)$ & $0.0214(6)$ \\
\hline N8 & $0.3906(2)$ & $0.5038(2)$ & $0.16787(9)$ & $0.0229(5)$ \\
\hline H8 & $0.437(3)$ & $0.547(2)$ & $0.1368(12)$ & $0.028^{*}$ \\
\hline C8A & $0.2622(3)$ & $0.4257(2)$ & $0.15110(11)$ & $0.0225(6)$ \\
\hline C9 & $0.1871(3)$ & $0.3838(2)$ & $0.08913(12)$ & $0.0268(6)$ \\
\hline H9 & 0.2220 & 0.4098 & 0.0497 & $0.032 *$ \\
\hline $\mathrm{C} 10$ & $0.0602(3)$ & 0.3033 & $0.08712(13)$ & $0.0299(7)$ \\
\hline H10 & 0.0070 & 0.2722 & 0.0461 & $0.036^{*}$ \\
\hline C11 & $0.0114(3)$ & 0.2683 & $0.14629(13)$ & $0.0283(6)$ \\
\hline $\mathrm{C} 12$ & 0.0805 & $0.3085(2)$ & $0.20795(12)$ & $0.0248(6)$ \\
\hline H12 & 0.0424 & 0.2828 & 0.2467 & $0.030^{*}$ \\
\hline C12A & $0.2112(2)$ & $0.3900(2)$ & $0.21103(11)$ & $0.0214(6)$ \\
\hline C12B & $0.3143(3)$ & $0.4501(2)$ & $0.26446(11)$ & $0.0201(6)$ \\
\hline C13 & 0.2988 & 0.2225 & $0.38597(11)$ & $0.0221(6)$ \\
\hline
\end{tabular}




$\begin{array}{lllll}\text { C14 } & 0.0434(3) & 0.1308(3) & 0.38115(13) & 0.0292(7) \\ \text { H14A } & 0.0642 & 0.0713 & 0.3480 & 0.044^{*} \\ \text { H14B } & -0.0707 & 0.1519 & 0.3735 & 0.044^{*} \\ \text { H14C } & 0.0730 & 0.0958 & 0.4254 & 0.044^{*} \\ \text { C15 } & 0.6182(3) & 0.2417(2) & 0.45594(11) & 0.0212(6) \\ \text { C16 } & 0.5946(3) & 0.1559(3) & 0.55942(12) & 0.0295(7) \\ \text { H16A } & 0.5529 & 0.0769 & 0.5422 & 0.044^{*} \\ \text { H16B } & 0.5509 & 0.1755 & 0.5995 & 0.044^{*} \\ \text { H16C } & 0.7118 & 0.1522 & 0.5700 & 0.044^{*} \\ \text { C17 } & 0.7970(3) & 0.4562(3) & 0.43429(11) & 0.0248(6) \\ \text { H17A } & 0.8687 & 0.5087 & 0.4131 & 0.030^{*} \\ \text { H17B } & 0.8583 & 0.3829 & 0.4514 & 0.030^{*} \\ \text { C18 } & 0.7454(3) & 0.5244(3) & 0.49167(13) & 0.0317(7) \\ \text { H18A } & 0.6843 & 0.5969 & 0.4750 & 0.048^{*} \\ \text { H18B } & 0.8404 & 0.5489 & 0.5230 & 0.048^{*} \\ \text { H18C } & 0.6782 & 0.4714 & 0.5140 & 0.048^{*}\end{array}$

Atomic displacement parameters $\left(\AA^{2}\right)$

\begin{tabular}{|c|c|c|c|c|c|c|}
\hline & $U^{11}$ & $U^{22}$ & $U^{33}$ & $U^{12}$ & $U^{13}$ & $U^{23}$ \\
\hline $\mathrm{F} 1$ & $0.0287(8)$ & $0.0431(12)$ & $0.0508(10)$ & $-0.0170(7)$ & $-0.0099(7)$ & $0.0069(8)$ \\
\hline $\mathrm{O} 1$ & $0.0152(8)$ & $0.0275(12)$ & $0.0328(10)$ & $0.0029(8)$ & $0.0016(7)$ & $0.0025(8)$ \\
\hline $\mathrm{O} 2$ & $0.0108(8)$ & $0.0301(12)$ & $0.0356(10)$ & $-0.0008(7)$ & $0.0034(7)$ & $0.0043(8)$ \\
\hline $\mathrm{O} 3$ & $0.0172(8)$ & $0.0318(12)$ & $0.0300(10)$ & $0.0066(7)$ & $0.0024(7)$ & $0.0008(8)$ \\
\hline $\mathrm{O} 4$ & $0.0215(9)$ & $0.0338(12)$ & $0.0189(9)$ & $0.0030(7)$ & $0.0052(6)$ & $0.0043(8)$ \\
\hline $\mathrm{C} 1$ & $0.0127(11)$ & $0.0284(17)$ & $0.0241(13)$ & $0.0018(10)$ & $0.0003(9)$ & $0.0018(11)$ \\
\hline $\mathrm{C} 2$ & $0.0133(11)$ & $0.0260(16)$ & $0.0195(12)$ & $-0.0001(10)$ & $0.0016(9)$ & $0.0000(10)$ \\
\hline $\mathrm{C} 3$ & $0.0147(11)$ & $0.0262(16)$ & $0.0181(11)$ & $0.0016(10)$ & $0.0020(8)$ & $-0.0007(10)$ \\
\hline N4 & $0.0135(9)$ & $0.0308(14)$ & $0.0194(10)$ & $-0.0041(9)$ & $0.0006(7)$ & $0.0001(9)$ \\
\hline $\mathrm{C} 5$ & $0.0147(11)$ & $0.0326(17)$ & $0.0198(12)$ & $0.0000(10)$ & $0.0018(9)$ & $-0.0001(11)$ \\
\hline C6 & $0.0165(12)$ & $0.0375(18)$ & $0.0243(13)$ & -0.0068 & $0.0005(9)$ & $0.0023(12)$ \\
\hline $\mathrm{C} 7$ & $0.0205(12)$ & $0.0309(17)$ & $0.0222(13)$ & $-0.0044(11)$ & $0.0004(10)$ & $0.0032(11)$ \\
\hline $\mathrm{C} 7 \mathrm{~A}$ & $0.0182(12)$ & $0.0251(16)$ & $0.0196(13)$ & $0.0021(10)$ & $-0.0007(9)$ & $0.0005(11)$ \\
\hline N8 & $0.0173(10)$ & $0.0313(15)$ & $0.0190(11)$ & $-0.0035(9)$ & $0.0000(8)$ & $0.0028(10)$ \\
\hline $\mathrm{C} 8 \mathrm{~A}$ & $0.0137(11)$ & $0.0286(17)$ & $0.0235(13)$ & $0.0018(10)$ & $-0.0016(9)$ & $0.0001(11)$ \\
\hline C9 & $0.0201(12)$ & $0.0321(19)$ & $0.0267(14)$ & $0.0026(11)$ & $0.0000(10)$ & $0.0002(12)$ \\
\hline $\mathrm{C} 10$ & $0.0194(12)$ & $0.0363(19)$ & $0.0295(14)$ & $0.0012(12)$ & $-0.0088(10)$ & $-0.0024(12)$ \\
\hline C11 & $0.0147(12)$ & $0.0284(18)$ & $0.0377(15)$ & $-0.0034(11)$ & $-0.0073(10)$ & $0.0043(13)$ \\
\hline $\mathrm{C} 12$ & $0.0150(11)$ & $0.0277(17)$ & $0.0299(14)$ & $0.0019(10)$ & $-0.0015(9)$ & $0.0073(12)$ \\
\hline $\mathrm{C} 12 \mathrm{~A}$ & $0.0122(11)$ & $0.0267(17)$ & $0.0237(13)$ & $0.0035(10)$ & $-0.0019(9)$ & $0.0048(11)$ \\
\hline $\mathrm{C} 12 \mathrm{~B}$ & $0.0143(11)$ & $0.0261(16)$ & $0.0193(12)$ & $0.0040(10)$ & $0.0009(9)$ & $0.0045(11)$ \\
\hline $\mathrm{C} 13$ & $0.0116(11)$ & $0.0356(19)$ & $0.0182(12)$ & $0.0015(11)$ & $0.0000(8)$ & $-0.0008(11)$ \\
\hline C14 & $0.0144(11)$ & $0.0300(18)$ & $0.0441(16)$ & $-0.0057(11)$ & $0.0076(10)$ & $0.0043(13)$ \\
\hline $\mathrm{C} 15$ & $0.0141(11)$ & $0.0280(17)$ & $0.0204(12)$ & $-0.0026(10)$ & $-0.0001(9)$ & $-0.0015(11)$ \\
\hline $\mathrm{C} 16$ & $0.0231(13)$ & $0.0402(19)$ & $0.0229(13)$ & $-0.0009(12)$ & $-0.0027(10)$ & $0.0095(12)$ \\
\hline $\mathrm{C} 17$ & $0.0159(11)$ & $0.0321(17)$ & $0.0248(13)$ & $-0.0033(11)$ & $-0.0013(9)$ & $0.0019(11)$ \\
\hline $\mathrm{C} 18$ & $0.0208(12)$ & $0.0424(19)$ & $0.0305(14)$ & $-0.0068(12)$ & $-0.0002(10)$ & $-0.0059(13)$ \\
\hline
\end{tabular}


Geometric parameters $\left(\AA,{ }^{\circ}\right)$

\begin{tabular}{|c|c|c|c|}
\hline $\mathrm{F} 1-\mathrm{C} 11$ & $1.383(3)$ & $\mathrm{C} 7 \mathrm{~A}-\mathrm{N} 8$ & $1.382(3)$ \\
\hline $\mathrm{O} 1-\mathrm{C} 13$ & $1.228(3)$ & $\mathrm{C} 7 \mathrm{~A}-\mathrm{C} 12 \mathrm{~B}$ & $1.390(3)$ \\
\hline $\mathrm{O} 2-\mathrm{C} 13$ & $1.352(3)$ & $\mathrm{N} 8-\mathrm{C} 8 \mathrm{~A}$ & $1.383(3)$ \\
\hline $\mathrm{O} 2-\mathrm{C} 14$ & $1.461(3)$ & $\mathrm{N} 8-\mathrm{H} 8$ & $0.93(3)$ \\
\hline $\mathrm{O} 3-\mathrm{C} 15$ & $1.213(3)$ & $\mathrm{C} 8 \mathrm{~A}-\mathrm{C} 9$ & $1.401(3)$ \\
\hline $\mathrm{O} 4-\mathrm{C} 15$ & $1.347(3)$ & $\mathrm{C} 8 \mathrm{~A}-\mathrm{C} 12 \mathrm{~A}$ & $1.425(3)$ \\
\hline $\mathrm{O} 4-\mathrm{C} 16$ & $1.451(3)$ & $\mathrm{C} 9-\mathrm{C} 10$ & $1.388(3)$ \\
\hline $\mathrm{C} 1-\mathrm{C} 12 \mathrm{~B}$ & $1.502(3)$ & $\mathrm{C} 9-\mathrm{H} 9$ & 0.9500 \\
\hline $\mathrm{C} 1-\mathrm{C} 2$ & $1.532(3)$ & $\mathrm{C} 10-\mathrm{C} 11$ & $1.400(4)$ \\
\hline $\mathrm{C} 1-\mathrm{H} 1 \mathrm{~A}$ & 0.9900 & $\mathrm{C} 10-\mathrm{H} 10$ & 0.9500 \\
\hline $\mathrm{C} 1-\mathrm{H} 1 \mathrm{~B}$ & 0.9900 & $\mathrm{C} 11-\mathrm{C} 12$ & $1.376(3)$ \\
\hline $\mathrm{C} 2-\mathrm{C} 3$ & $1.366(3)$ & $\mathrm{C} 12-\mathrm{C} 12 \mathrm{~A}$ & $1.417(3)$ \\
\hline $\mathrm{C} 2-\mathrm{C} 13$ & $1.487(4)$ & $\mathrm{C} 12-\mathrm{H} 12$ & 0.9500 \\
\hline $\mathrm{C} 3-\mathrm{N} 4$ & $1.407(3)$ & $\mathrm{C} 12 \mathrm{~A}-\mathrm{C} 12 \mathrm{~B}$ & $1.442(3)$ \\
\hline $\mathrm{C} 3-\mathrm{C} 15$ & $1.530(3)$ & $\mathrm{C} 14-\mathrm{H} 14 \mathrm{~A}$ & 0.9800 \\
\hline $\mathrm{N} 4-\mathrm{C} 17$ & $1.475(3)$ & C14-H14B & 0.9800 \\
\hline $\mathrm{N} 4-\mathrm{C} 5$ & $1.488(3)$ & $\mathrm{C} 14-\mathrm{H} 14 \mathrm{C}$ & 0.9800 \\
\hline $\mathrm{C} 5-\mathrm{C} 6$ & $1.531(4)$ & $\mathrm{C} 16-\mathrm{H} 16 \mathrm{~A}$ & 0.9800 \\
\hline $\mathrm{C} 5-\mathrm{H} 5 \mathrm{~A}$ & 0.9900 & C16-H16B & 0.9800 \\
\hline C5-H5B & 0.9900 & $\mathrm{C} 16-\mathrm{H} 16 \mathrm{C}$ & 0.9800 \\
\hline $\mathrm{C} 6-\mathrm{C} 7$ & $1.542(3)$ & $\mathrm{C} 17-\mathrm{C} 18$ & $1.520(4)$ \\
\hline $\mathrm{C} 6-\mathrm{H} 6 \mathrm{~A}$ & 0.9900 & C17-H17A & 0.9900 \\
\hline C6-H6B & 0.9900 & C17-H17B & 0.9900 \\
\hline $\mathrm{C} 7-\mathrm{C} 7 \mathrm{~A}$ & $1.499(3)$ & $\mathrm{C} 18-\mathrm{H} 18 \mathrm{~A}$ & 0.9800 \\
\hline C7-H7A & 0.9900 & $\mathrm{C} 18-\mathrm{H} 18 \mathrm{~B}$ & 0.9800 \\
\hline $\mathrm{C} 7-\mathrm{H} 7 \mathrm{~B}$ & 0.9900 & $\mathrm{C} 18-\mathrm{H} 18 \mathrm{C}$ & 0.9800 \\
\hline $\mathrm{C} 13-\mathrm{O} 2-\mathrm{C} 14$ & $114.88(19)$ & $\mathrm{C} 8 \mathrm{~A}-\mathrm{C} 9-\mathrm{H} 9$ & 121.0 \\
\hline $\mathrm{C} 15-\mathrm{O} 4-\mathrm{C} 16$ & $115.13(19)$ & $\mathrm{C} 9-\mathrm{C} 10-\mathrm{C} 11$ & $119.3(2)$ \\
\hline $\mathrm{C} 12 \mathrm{~B}-\mathrm{C} 1-\mathrm{C} 2$ & $118.4(2)$ & $\mathrm{C} 9-\mathrm{C} 10-\mathrm{H} 10$ & 120.4 \\
\hline $\mathrm{C} 12 \mathrm{~B}-\mathrm{C} 1-\mathrm{H} 1 \mathrm{~A}$ & 107.7 & $\mathrm{C} 11-\mathrm{C} 10-\mathrm{H} 10$ & 120.4 \\
\hline $\mathrm{C} 2-\mathrm{C} 1-\mathrm{H} 1 \mathrm{~A}$ & 107.7 & $\mathrm{C} 12-\mathrm{C} 11-\mathrm{F} 1$ & $118.4(2)$ \\
\hline $\mathrm{C} 12 \mathrm{~B}-\mathrm{C} 1-\mathrm{H} 1 \mathrm{~B}$ & 107.7 & $\mathrm{C} 12-\mathrm{C} 11-\mathrm{C} 10$ & $124.6(2)$ \\
\hline $\mathrm{C} 2-\mathrm{C} 1-\mathrm{H} 1 \mathrm{~B}$ & 107.7 & $\mathrm{~F} 1-\mathrm{C} 11-\mathrm{C} 10$ & $117.0(2)$ \\
\hline $\mathrm{H} 1 \mathrm{~A}-\mathrm{C} 1-\mathrm{H} 1 \mathrm{~B}$ & 107.1 & $\mathrm{C} 11-\mathrm{C} 12-\mathrm{C} 12 \mathrm{~A}$ & $117.0(2)$ \\
\hline $\mathrm{C} 3-\mathrm{C} 2-\mathrm{C} 13$ & $117.2(2)$ & $\mathrm{C} 11-\mathrm{C} 12-\mathrm{H} 12$ & 121.5 \\
\hline $\mathrm{C} 3-\mathrm{C} 2-\mathrm{C} 1$ & $122.2(2)$ & $\mathrm{C} 12 \mathrm{~A}-\mathrm{C} 12-\mathrm{H} 12$ & 121.5 \\
\hline $\mathrm{C} 13-\mathrm{C} 2-\mathrm{C} 1$ & $120.61(19)$ & $\mathrm{C} 12-\mathrm{C} 12 \mathrm{~A}-\mathrm{C} 8 \mathrm{~A}$ & $118.8(2)$ \\
\hline $\mathrm{C} 2-\mathrm{C} 3-\mathrm{N} 4$ & $122.4(2)$ & $\mathrm{C} 12-\mathrm{C} 12 \mathrm{~A}-\mathrm{C} 12 \mathrm{~B}$ & $133.8(2)$ \\
\hline $\mathrm{C} 2-\mathrm{C} 3-\mathrm{C} 15$ & $120.9(2)$ & $\mathrm{C} 8 \mathrm{~A}-\mathrm{C} 12 \mathrm{~A}-\mathrm{C} 12 \mathrm{~B}$ & $107.3(2)$ \\
\hline $\mathrm{N} 4-\mathrm{C} 3-\mathrm{C} 15$ & $116.65(18)$ & $\mathrm{C} 7 \mathrm{~A}-\mathrm{C} 12 \mathrm{~B}-\mathrm{C} 12 \mathrm{~A}$ & $106.40(19)$ \\
\hline $\mathrm{C} 3-\mathrm{N} 4-\mathrm{C} 17$ & $117.83(18)$ & $\mathrm{C} 7 \mathrm{~A}-\mathrm{C} 12 \mathrm{~B}-\mathrm{C} 1$ & $125.5(2)$ \\
\hline $\mathrm{C} 3-\mathrm{N} 4-\mathrm{C} 5$ & $115.06(19)$ & $\mathrm{C} 12 \mathrm{~A}-\mathrm{C} 12 \mathrm{~B}-\mathrm{C} 1$ & $128.1(2)$ \\
\hline $\mathrm{C} 17-\mathrm{N} 4-\mathrm{C} 5$ & $113.43(16)$ & $\mathrm{O} 1-\mathrm{C} 13-\mathrm{O} 2$ & $122.0(2)$ \\
\hline $\mathrm{N} 4-\mathrm{C} 5-\mathrm{C} 6$ & $113.7(2)$ & $\mathrm{O} 1-\mathrm{C} 13-\mathrm{C} 2$ & $124.4(2)$ \\
\hline $\mathrm{N} 4-\mathrm{C} 5-\mathrm{H} 5 \mathrm{~A}$ & 108.8 & $\mathrm{O} 2-\mathrm{C} 13-\mathrm{C} 2$ & $113.6(2)$ \\
\hline
\end{tabular}




\begin{tabular}{|c|c|c|c|}
\hline $\mathrm{C} 6-\mathrm{C} 5-\mathrm{H} 5 \mathrm{~A}$ & 108.8 & $\mathrm{O} 2-\mathrm{C} 14-\mathrm{H} 14 \mathrm{~A}$ & 109.5 \\
\hline $\mathrm{N} 4-\mathrm{C} 5-\mathrm{H} 5 \mathrm{~B}$ & 108.8 & $\mathrm{O} 2-\mathrm{C} 14-\mathrm{H} 14 \mathrm{~B}$ & 109.5 \\
\hline $\mathrm{C} 6-\mathrm{C} 5-\mathrm{H} 5 \mathrm{~B}$ & 108.8 & $\mathrm{H} 14 \mathrm{~A}-\mathrm{C} 14-\mathrm{H} 14 \mathrm{~B}$ & 109.5 \\
\hline $\mathrm{H} 5 \mathrm{~A}-\mathrm{C} 5-\mathrm{H} 5 \mathrm{~B}$ & 107.7 & $\mathrm{O} 2-\mathrm{C} 14-\mathrm{H} 14 \mathrm{C}$ & 109.5 \\
\hline $\mathrm{C} 5-\mathrm{C} 6-\mathrm{C} 7$ & $113.24(19)$ & $\mathrm{H} 14 \mathrm{~A}-\mathrm{C} 14-\mathrm{H} 14 \mathrm{C}$ & 109.5 \\
\hline $\mathrm{C} 5-\mathrm{C} 6-\mathrm{H} 6 \mathrm{~A}$ & 108.9 & $\mathrm{H} 14 \mathrm{~B}-\mathrm{C} 14-\mathrm{H} 14 \mathrm{C}$ & 109.5 \\
\hline $\mathrm{C} 7-\mathrm{C} 6-\mathrm{H} 6 \mathrm{~A}$ & 108.9 & $\mathrm{O} 3-\mathrm{C} 15-\mathrm{O} 4$ & $124.7(2)$ \\
\hline $\mathrm{C} 5-\mathrm{C} 6-\mathrm{H} 6 \mathrm{~B}$ & 108.9 & $\mathrm{O} 3-\mathrm{C} 15-\mathrm{C} 3$ & $123.6(2)$ \\
\hline $\mathrm{C} 7-\mathrm{C} 6-\mathrm{H} 6 \mathrm{~B}$ & 108.9 & $\mathrm{O} 4-\mathrm{C} 15-\mathrm{C} 3$ & $111.68(19)$ \\
\hline $\mathrm{H} 6 \mathrm{~A}-\mathrm{C} 6-\mathrm{H} 6 \mathrm{~B}$ & 107.7 & $\mathrm{O} 4-\mathrm{C} 16-\mathrm{H} 16 \mathrm{~A}$ & 109.5 \\
\hline $\mathrm{C} 7 \mathrm{~A}-\mathrm{C} 7-\mathrm{C} 6$ & $111.7(2)$ & $\mathrm{O} 4-\mathrm{C} 16-\mathrm{H} 16 \mathrm{~B}$ & 109.5 \\
\hline $\mathrm{C} 7 \mathrm{~A}-\mathrm{C} 7-\mathrm{H} 7 \mathrm{~A}$ & 109.3 & $\mathrm{H} 16 \mathrm{~A}-\mathrm{C} 16-\mathrm{H} 16 \mathrm{~B}$ & 109.5 \\
\hline $\mathrm{C} 6-\mathrm{C} 7-\mathrm{H} 7 \mathrm{~A}$ & 109.3 & $\mathrm{O} 4-\mathrm{C} 16-\mathrm{H} 16 \mathrm{C}$ & 109.5 \\
\hline $\mathrm{C} 7 \mathrm{~A}-\mathrm{C} 7-\mathrm{H} 7 \mathrm{~B}$ & 109.3 & $\mathrm{H} 16 \mathrm{~A}-\mathrm{C} 16-\mathrm{H} 16 \mathrm{C}$ & 109.5 \\
\hline $\mathrm{C} 6-\mathrm{C} 7-\mathrm{H} 7 \mathrm{~B}$ & 109.3 & $\mathrm{H} 16 \mathrm{~B}-\mathrm{C} 16-\mathrm{H} 16 \mathrm{C}$ & 109.5 \\
\hline $\mathrm{H} 7 \mathrm{~A}-\mathrm{C} 7-\mathrm{H} 7 \mathrm{~B}$ & 107.9 & $\mathrm{~N} 4-\mathrm{C} 17-\mathrm{C} 18$ & $112.19(18)$ \\
\hline $\mathrm{N} 8-\mathrm{C} 7 \mathrm{~A}-\mathrm{C} 12 \mathrm{~B}$ & $109.5(2)$ & N4-C17-H17A & 109.2 \\
\hline $\mathrm{N} 8-\mathrm{C} 7 \mathrm{~A}-\mathrm{C} 7$ & $120.7(2)$ & $\mathrm{C} 18-\mathrm{C} 17-\mathrm{H} 17 \mathrm{~A}$ & 109.2 \\
\hline $\mathrm{C} 12 \mathrm{~B}-\mathrm{C} 7 \mathrm{~A}-\mathrm{C} 7$ & $129.5(2)$ & $\mathrm{N} 4-\mathrm{C} 17-\mathrm{H} 17 \mathrm{~B}$ & 109.2 \\
\hline $\mathrm{C} 7 \mathrm{~A}-\mathrm{N} 8-\mathrm{C} 8 \mathrm{~A}$ & $109.62(19)$ & $\mathrm{C} 18-\mathrm{C} 17-\mathrm{H} 17 \mathrm{~B}$ & 109.2 \\
\hline $\mathrm{C} 7 \mathrm{~A}-\mathrm{N} 8-\mathrm{H} 8$ & $126.5(15)$ & $\mathrm{H} 17 \mathrm{~A}-\mathrm{C} 17-\mathrm{H} 17 \mathrm{~B}$ & 107.9 \\
\hline $\mathrm{C} 8 \mathrm{~A}-\mathrm{N} 8-\mathrm{H} 8$ & $123.3(15)$ & $\mathrm{C} 17-\mathrm{C} 18-\mathrm{H} 18 \mathrm{~A}$ & 109.5 \\
\hline $\mathrm{N} 8-\mathrm{C} 8 \mathrm{~A}-\mathrm{C} 9$ & $130.4(2)$ & $\mathrm{C} 17-\mathrm{C} 18-\mathrm{H} 18 \mathrm{~B}$ & 109.5 \\
\hline $\mathrm{N} 8-\mathrm{C} 8 \mathrm{~A}-\mathrm{C} 12 \mathrm{~A}$ & $107.2(2)$ & $\mathrm{H} 18 \mathrm{~A}-\mathrm{C} 18-\mathrm{H} 18 \mathrm{~B}$ & 109.5 \\
\hline $\mathrm{C} 9-\mathrm{C} 8 \mathrm{~A}-\mathrm{C} 12 \mathrm{~A}$ & $122.4(2)$ & $\mathrm{C} 17-\mathrm{C} 18-\mathrm{H} 18 \mathrm{C}$ & 109.5 \\
\hline $\mathrm{C} 10-\mathrm{C} 9-\mathrm{C} 8 \mathrm{~A}$ & $117.9(2)$ & $\mathrm{H} 18 \mathrm{~A}-\mathrm{C} 18-\mathrm{H} 18 \mathrm{C}$ & 109.5 \\
\hline $\mathrm{C} 10-\mathrm{C} 9-\mathrm{H} 9$ & 121.0 & $\mathrm{H} 18 \mathrm{~B}-\mathrm{C} 18-\mathrm{H} 18 \mathrm{C}$ & 109.5 \\
\hline $\mathrm{C} 12 \mathrm{~B}-\mathrm{C} 1-\mathrm{C} 2-\mathrm{C} 3$ & $88.1(3)$ & $\mathrm{N} 8-\mathrm{C} 8 \mathrm{~A}-\mathrm{C} 12 \mathrm{~A}-\mathrm{C} 12$ & $-179.6(2)$ \\
\hline $\mathrm{C} 12 \mathrm{~B}-\mathrm{C} 1-\mathrm{C} 2-\mathrm{C} 13$ & $-93.4(3)$ & $\mathrm{C} 9-\mathrm{C} 8 \mathrm{~A}-\mathrm{C} 12 \mathrm{~A}-\mathrm{C} 12$ & $0.4(4)$ \\
\hline $\mathrm{C} 13-\mathrm{C} 2-\mathrm{C} 3-\mathrm{N} 4$ & $163.5(2)$ & $\mathrm{N} 8-\mathrm{C} 8 \mathrm{~A}-\mathrm{C} 12 \mathrm{~A}-\mathrm{C} 12 \mathrm{~B}$ & $0.1(3)$ \\
\hline $\mathrm{C} 1-\mathrm{C} 2-\mathrm{C} 3-\mathrm{N} 4$ & $-18.0(3)$ & $\mathrm{C} 9-\mathrm{C} 8 \mathrm{~A}-\mathrm{C} 12 \mathrm{~A}-\mathrm{C} 12 \mathrm{~B}$ & $-179.9(2)$ \\
\hline $\mathrm{C} 13-\mathrm{C} 2-\mathrm{C} 3-\mathrm{C} 15$ & $-14.3(3)$ & $\mathrm{N} 8-\mathrm{C} 7 \mathrm{~A}-\mathrm{C} 12 \mathrm{~B}-\mathrm{C} 12 \mathrm{~A}$ & $-0.9(3)$ \\
\hline $\mathrm{C} 1-\mathrm{C} 2-\mathrm{C} 3-\mathrm{C} 15$ & $164.2(2)$ & $\mathrm{C} 7-\mathrm{C} 7 \mathrm{~A}-\mathrm{C} 12 \mathrm{~B}-\mathrm{C} 12 \mathrm{~A}$ & $-174.2(2)$ \\
\hline $\mathrm{C} 2-\mathrm{C} 3-\mathrm{N} 4-\mathrm{C} 17$ & $150.5(2)$ & $\mathrm{N} 8-\mathrm{C} 7 \mathrm{~A}-\mathrm{C} 12 \mathrm{~B}-\mathrm{C} 1$ & $179.6(2)$ \\
\hline $\mathrm{C} 15-\mathrm{C} 3-\mathrm{N} 4-\mathrm{C} 17$ & $-31.6(3)$ & $\mathrm{C} 7-\mathrm{C} 7 \mathrm{~A}-\mathrm{C} 12 \mathrm{~B}-\mathrm{C} 1$ & $6.3(4)$ \\
\hline $\mathrm{C} 2-\mathrm{C} 3-\mathrm{N} 4-\mathrm{C} 5$ & $-71.5(3)$ & $\mathrm{C} 12-\mathrm{C} 12 \mathrm{~A}-\mathrm{C} 12 \mathrm{~B}-\mathrm{C} 7 \mathrm{~A}$ & $-179.9(2)$ \\
\hline $\mathrm{C} 15-\mathrm{C} 3-\mathrm{N} 4-\mathrm{C} 5$ & $106.4(2)$ & $\mathrm{C} 8 \mathrm{~A}-\mathrm{C} 12 \mathrm{~A}-\mathrm{C} 12 \mathrm{~B}-\mathrm{C} 7 \mathrm{~A}$ & $0.5(3)$ \\
\hline $\mathrm{C} 3-\mathrm{N} 4-\mathrm{C} 5-\mathrm{C} 6$ & $141.0(2)$ & $\mathrm{C} 12-\mathrm{C} 12 \mathrm{~A}-\mathrm{C} 12 \mathrm{~B}-\mathrm{C} 1$ & $-0.4(4)$ \\
\hline $\mathrm{C} 17-\mathrm{N} 4-\mathrm{C} 5-\mathrm{C} 6$ & $-79.1(2)$ & $\mathrm{C} 8 \mathrm{~A}-\mathrm{C} 12 \mathrm{~A}-\mathrm{C} 12 \mathrm{~B}-\mathrm{C} 1$ & $-180.0(2)$ \\
\hline $\mathrm{N} 4-\mathrm{C} 5-\mathrm{C} 6-\mathrm{C} 7$ & $-58.9(2)$ & $\mathrm{C} 2-\mathrm{C} 1-\mathrm{C} 12 \mathrm{~B}-\mathrm{C} 7 \mathrm{~A}$ & $-98.2(3)$ \\
\hline $\mathrm{C} 5-\mathrm{C} 6-\mathrm{C} 7-\mathrm{C} 7 \mathrm{~A}$ & $-54.3(3)$ & $\mathrm{C} 2-\mathrm{C} 1-\mathrm{C} 12 \mathrm{~B}-\mathrm{C} 12 \mathrm{~A}$ & $82.3(3)$ \\
\hline $\mathrm{C} 6-\mathrm{C} 7-\mathrm{C} 7 \mathrm{~A}-\mathrm{N} 8$ & $-83.2(3)$ & $\mathrm{C} 14-\mathrm{O} 2-\mathrm{C} 13-\mathrm{O} 1$ & $-2.1(3)$ \\
\hline $\mathrm{C} 6-\mathrm{C} 7-\mathrm{C} 7 \mathrm{~A}-\mathrm{C} 12 \mathrm{~B}$ & $89.4(3)$ & $\mathrm{C} 14-\mathrm{O} 2-\mathrm{C} 13-\mathrm{C} 2$ & $176.11(18)$ \\
\hline $\mathrm{C} 12 \mathrm{~B}-\mathrm{C} 7 \mathrm{~A}-\mathrm{N} 8-\mathrm{C} 8 \mathrm{~A}$ & $1.0(3)$ & $\mathrm{C} 3-\mathrm{C} 2-\mathrm{C} 13-\mathrm{O} 1$ & $-21.2(3)$ \\
\hline $\mathrm{C} 7-\mathrm{C} 7 \mathrm{~A}-\mathrm{N} 8-\mathrm{C} 8 \mathrm{~A}$ & $174.9(2)$ & $\mathrm{C} 1-\mathrm{C} 2-\mathrm{C} 13-\mathrm{O} 1$ & $160.2(2)$ \\
\hline $\mathrm{C} 7 \mathrm{~A}-\mathrm{N} 8-\mathrm{C} 8 \mathrm{~A}-\mathrm{C} 9$ & $179.3(3)$ & $\mathrm{C} 3-\mathrm{C} 2-\mathrm{C} 13-\mathrm{O} 2$ & $160.6(2)$ \\
\hline $\mathrm{C} 7 \mathrm{~A}-\mathrm{N} 8-\mathrm{C} 8 \mathrm{~A}-\mathrm{C} 12 \mathrm{~A}$ & $-0.6(3)$ & $\mathrm{C} 1-\mathrm{C} 2-\mathrm{C} 13-\mathrm{O} 2$ & $-18.0(3)$ \\
\hline
\end{tabular}




$\begin{array}{llll}\mathrm{N} 8-\mathrm{C} 8 \mathrm{~A}-\mathrm{C} 9-\mathrm{C} 10 & 179.1(2) & \mathrm{C} 16-\mathrm{O} 4-\mathrm{C} 15-\mathrm{O} 3 & -8.8(3) \\ \mathrm{C} 12 \mathrm{~A}-\mathrm{C} 8 \mathrm{~A}-\mathrm{C} 9-\mathrm{C} 10 & -1.0(4) & \mathrm{C} 16-\mathrm{O} 4-\mathrm{C} 15-\mathrm{C} 3 & 174.42(19) \\ \mathrm{C} 8 \mathrm{~A}-\mathrm{C} 9-\mathrm{C} 10-\mathrm{C} 11 & 0.7(4) & \mathrm{C} 2-\mathrm{C} 3-\mathrm{C} 15-\mathrm{O} 3 & 122.1(3) \\ \mathrm{C} 9-\mathrm{C} 10-\mathrm{C} 11-\mathrm{C} 12 & 0.2(4) & \mathrm{N} 4-\mathrm{C} 3-\mathrm{C} 15-\mathrm{O} 3 & -55.9(3) \\ \mathrm{C} 9-\mathrm{C} 10-\mathrm{C} 11-\mathrm{F} 1 & \mathrm{C} 2-\mathrm{C} 3-\mathrm{C} 15-\mathrm{O} 4 & 121.0(2) \\ \mathrm{F} 1-\mathrm{C} 11-\mathrm{C} 12-\mathrm{C} 12 \mathrm{~A} & 178.0(2) & \mathrm{N} 4-\mathrm{C} 3-\mathrm{C} 15-\mathrm{O} 4 & -64.2(3) \\ \mathrm{C} 10-\mathrm{C} 11-\mathrm{C} 12-\mathrm{C} 12 \mathrm{~A} & -0.7(4) & \mathrm{C} 3-\mathrm{N} 4-\mathrm{C} 17-\mathrm{C} 18 & 157.2(2) \\ \mathrm{C} 11-\mathrm{C} 12-\mathrm{C} 12 \mathrm{~A}-\mathrm{C} 8 \mathrm{~A} & 0.4(3) & \mathrm{C} 5-\mathrm{N} 4-\mathrm{C} 17-\mathrm{C} 18 & \\ \mathrm{C} 11-\mathrm{C} 12-\mathrm{C} 12 \mathrm{~A}-\mathrm{C} 12 \mathrm{~B} & -179.2(3) & & \end{array}$

Hydrogen-bond geometry ( $\left.\AA,{ }^{\circ}\right)$

\begin{tabular}{lllll}
\hline$D-\mathrm{H} \cdots A$ & $D-\mathrm{H}$ & $\mathrm{H} \cdots A$ & $D \cdots A$ & $D-\mathrm{H} \cdots A$ \\
\hline $\mathrm{N} 8-\mathrm{H} 8 \cdots \mathrm{O} 1^{\mathrm{i}}$ & $0.93(3)$ & $2.17(3)$ & $3.025(3)$ & $153(2)$ \\
\hline
\end{tabular}

Symmetry code: (i) $-x+1, y+1 / 2,-z+1 / 2$. 